RESEARCH

Turkish Journal of Geriatrics

DOI: $10.31086 /$ tigeri.2020.173

2020; 23(3): 362-373

- Vermi DEĞERLI' ${ }^{1}$ (D)

- Tanzer KORKMAZ² (D)

CORRESPONDANCE

Vermi DEĞERLi

University of Health Sciences, Bozyaka

Teaching and Research Hospital, Department of

Emergency Medicine, İzmir, Turkey

Phone: +905072334186

e-mail: vermidegerli@yahoo.com

Received: Jul 17, 2020

Accepted: Aug 12, 2020

\section{CHARACTERISTICS AND OUTCOMES IN ELDERLY PATIENTS WITH DYSPNEA ARRIVING BY AMBULANCE AT THE EMERGENCY DEPARTMENT OF A TERTIARY HOSPITAL}

\section{Abstract}

Introduction: We investigated the characteristics and outcomes in elderly patients with non-traumatic dyspnea who arrived at the emergency department by ambulance.

Materials and Method: This retrospective descriptive study included patients aged $\geq 65$ years, who arrived at the emergency department by ambulance with a chief complaint respiratory distress, between January 1 and December 31, 2018. We recorded age, sex, time and day of admission, season, length of stay in the emergency department, comorbidities, interventions, treatments, consultations, emergency department diagnoses, and patient outcomes.

Results: Of the 4089 elderly patients who arrived by ambulance, 16\% ( $n=655$ ) had respiratory distress. Mean patient ages was $78.23 \pm 0.30(65-98)$ years and $52.1 \%$ were female. Most admissions occurred in winter (30.7\%). The most common comorbidities included hypertension (74.4\%), chronic obstructive pulmonary disease $(48.1 \%)$, heart failure (45.8\%), and coronary artery disease (37.9\%). The most common emergency department diagnoses included chronic obstructive pulmonary disease exacerbation (32.6\%), pneumonia (31.1\%), heart failure $(26.1 \%)$, and hypertensive pulmonary edema ( $8 \%)$. The consultation rate was $65 \%$. Notably, $47.3 \%$ of patients were discharged from the emergency department, $6.4 \%$ were admitted to wards, and $34.5 \%$ were admitted to the intensive care unit. The inhospital mortality rate was 3.3\%.

Conclusions: The prevalence of dyspnea in elderly patients arriving at the emergency department by ambulance was higher in our study than in previous studies. We observed a high hospitalization rate with most admissions to the intensive care unit. Most patients were diagnosed with chronic respiratory and cardiac diseases.

Keywords: Dyspnea; Emergency service; Hospital; Ambulances; Geriatrics
1 University of Health Sciences, Bozyaka Teaching and Research Hospital, Department of Emergency Medicine, İzmir, Turkey

2 Cigli Teaching and Research Hospital, Department of Emergency Medicine, Izmir, Turkey 


\section{INTRODUCTION}

An increasing life expectancy, better living conditions and availability of healthcare services have resulted in rapid growth in the elderly population worldwide, as well as in Turkey. The percentage share of elderly individuals $>10 \%$ of the total population indicates an aging population. The percentage of the elderly population in Turkey is comparatively lower than that in many other countries; however, this figure is significantly high numerically. Reportedly, the elderly population (aged $\geq 65$ years) represented $8 \%$ and $8.8 \%$ of Turkey's total population in 2014 and 2018, respectively, and this rate is estimated to increase to $10.2 \%$ by 2023 and $12.9 \%$ by 2030 (1).

Little is known about the etiology, epidemiology, and outcome of dyspneic patients presenting to the EDs worldwide. Dyspnea, which is associated with a large number of ward and intensive care unit (ICU) admission, is the one of the most common cause that necessitates emergency departments (EDs) visit among elderly patients; these patients usually arrive at EDs by ambulance $(2,3)$. Recent studies have reported that the rate of ED arrival by ambulance secondary to dyspnea was approximately $12 \%$ in the USA, $5 \%$ in the AsiaPacific region, 6\% in Switzerland, 8\% in China, and $3.6 \%$ in Nepal $(4,5)$. In Turkey, in a recent study conducted by Dundar et al., it has been reported that the most common presenting symptom of elderly patients admitting to the ED is dyspnea (6). To our knowledge, no Turkish study has described the prevalence, epidemiology, etiology, diagnosis, treatment modalities, length of stay (LOS) in the ED and outcomes in elderly patients who present to EDs with a chief complaint of dyspnea.

Understanding the characteristics of elderly patients with dyspnea who arrive at the ED by ambulance, the range of diagnoses and their outcome are important for understanding the challenges facing emergency clinicians and for planning training and services.

This study aimed to describe the epidemiology in elderly patients with dyspnea who arrived at the ED by ambulance, to understand how it is investigated and treated and its outcome.

\section{MATERIALS AND METHODS}

This retrospective descriptive study was performed at the ED of the Bozyaka Training and Research Hospital of the University of Health Sciences, which is a tertiary ED with a 580bed capacity and a resident training program. We recorded approximately 190,000 patient admissions between January 1 and December 31, 2018. Patients aged $\geq 65$ years, who arrived by ambulance (112 emergency medical services) with the chief complaint shortness of breath were included in the study. For patients with repeated ED arrivals within the study period, we considered only the first arrival for this study. Patients with trauma-induced dyspnea and those aged $<65$ years were excluded from the study.

Data were obtained from the hospital information management system. We recorded the following information: age, sex, mode of arrival (via field or ambulance from other hospitals), time of arrival, day of arrival, season, length of stay (LOS) in the ED (defined as the interval [in hours] between arrival and ED discharge or hospital admission), comorbidities, investigations performed (laboratory tests, and imaging, among other testing), treatment in the ED, consultations, ED diagnosis (diagnosis after ED management), and ED outcomes in patients, The ED diagnoses were documented in all patients by the treating emergency physicians based on the International Statistical Classification of Diseases 10th Revision code.

Patients were classified into older and oldestold group. With regard to geriatric age groups, the World Health Organization categorizes older individuals as those aged between 65 and 79 years and the oldest-old as those aged $\geq 80$ years (7).

This study was approved by the Ethical Committee of the aforementioned hospital. Formal sample size calculation was not performed 
Table 1. Distribution of characteristics of the study population according to sex

\begin{tabular}{|c|c|c|c|}
\hline & Total & Females & Males \\
\hline \multicolumn{4}{|l|}{ Age (years) } \\
\hline Mean \pm SD (min-max) & $78.23 \pm 0.30$ (65-98) & $79.15 \pm 0.40$ (65-98) & $77.23 \pm 0.44(65-96)$ \\
\hline Older $(<80)(n, \%)$ & $372(56.8)$ & $184(49.5)$ & $188(50.5)$ \\
\hline Oldest-old ( $\geq 80)(n, \%)$ & $283(43.2)$ & $157(55.5)$ & $126(44.5)$ \\
\hline Gender (n, \%) & $655(100.0)$ & $341(52.1)$ & 314 (47.9) \\
\hline \multicolumn{4}{|l|}{ Season (n, \%) } \\
\hline Spring & $152(23.2)$ & $72(47.4)$ & $80(52.6)$ \\
\hline Summer & $130(19.8)$ & $79(60.8)$ & $51(39.2)$ \\
\hline Autumn & $172(26.3)$ & $81(47.1)$ & $91(52.9)$ \\
\hline Winter & $201(30.7)$ & $109(54.2)$ & $92(45.8)$ \\
\hline \multicolumn{4}{|c|}{ Type of admission ( $n, \%$ ) } \\
\hline Ambulance (field) & $629(96.0)$ & $328(52.1)$ & $301(47.9)$ \\
\hline Ambulance (hospital) & $26(4.0)$ & $13(50.0)$ & $13(50.0)$ \\
\hline \multicolumn{4}{|l|}{ Time of admission(n, \%) } \\
\hline $08.00-16.59 \mathrm{~h}$ & $240(36.6)$ & $116(48.3)$ & $124(36.6)$ \\
\hline $17.00-23.59 \mathrm{~h}$ & $243(37.1)$ & $139(57.2)$ & $243(37.1)$ \\
\hline $24.00-07.59 \mathrm{~h}$ & $172(26.3)$ & $86(50.0)$ & $172(26.3)$ \\
\hline \multicolumn{4}{|l|}{ Day of admission(n,\%) } \\
\hline Weekdays & $470(71.8)$ & 250 (53.2) & $220(46.89)$ \\
\hline Weekends & $185(28.2)$ & $91(49.2)$ & $94(50.8)$ \\
\hline
\end{tabular}

considering the descriptive nature of this study. Statistical analysis was performed using the IBM SPSS Statistics software, version 20.0 (Armonk, NY, USA). We used descriptive statistics including frequencies, percentages, means \pm standart deviation (min-max), or median (interquartile range $[I Q R], 95 \%$ confidence interval $[\mathrm{CI}])$. Data were compared based on age groups and sex group. The Mann-Whitney $U$ and the chisquare tests were used to compare continuous and categorical variables, respectively. $\mathrm{P}<0.05$ was considered statistically significant.

\section{RESULTS}

We observed that during the study period, 4089 elderly patients arrived at the ED by ambulance. Of these, 655 (16.01\%) were elderly patients who presented with shortness of breath. Mean patient age was 78.23 \pm 0.30 (65-98) years, 341 (52.1\%) were female, and 283 (43.20\%) were categorized as oldest-old patients. The admission rate was higher in winter (30.7\%) and less in summer (19.8\%). Only $4 \%$ of the 655 patients included in this study were referred to our hospital from other hospitals for further evaluation and treatment. The minimum number of admissions was recorded on weekends 
and between 24.00 and 07.59 hours. No statistically significant difference was identified between the age, season, admission (field/hospital), time of admission, and day of admission of the patients according to sex $(\mathrm{P}>0.05)$ (Table 1$)$.

The median LOS in the ED was 7.66 hours (IQR: 7.50, 95\% Cl [8.99-10.18]) for all patients. The median LOS of the older and oldest-old patients was 7.41 hours (IQR: $8.80,95 \% \mathrm{Cl}$ [8.81-10.53]) and 7.83 hours (IQR: $7.00,95 \% \mathrm{Cl}$ [8.69-10.25]), respectively. The Mann-Whitney $U$ test showed no significant difference between patient age groups and the LOS in the ED $(U=54.705, P=0.389)$.

Table 2 shows the distribution of comorbidities, imaging studies performed, interventions, treatment, and consultations of the elderly patients according to age groups. The most frequent comorbidities included hypertension (74.4\%), chronic obstructive pulmonary disease (COPD) (48.1\%), heart failure (HF) (45.8\%), and coronary artery diseases (37.9\%). COPD and diabetes mellitus were more common in older patients. Only $5.2 \% \quad(n=34)$ of the patients did not show comorbidities. No imaging was performed in $9.1 \%(n=60)$ of the patients. Chest radiography and thoracic computed tomography (CT) were the most common imaging modalities used in older patients, echocardiography and abdominal CT in oldest-old patients. Non-invasive ventilation was the most common interventions performed; however, no significant difference was observed between older and oldestold group in interventions performed. Other interventions included pleurocan catheterization $(n=3)$, tube thoracostomy $(n=6)$, paracentesis $(n=1)$, administration of blood products $(n=21)$, hemodialysis $(n=2)$, transcutaneous pacemaker $(n=1)$, and tracheostomy tube inner cannula changes $(n=2)$. Notably, 3.5\% $(n=23)$ of the patients did not receive any treatment. Diuretic treatment was commonly administered to older patients. In this study, 35.1\% ( $n=230)$ patients did not undergo any consultations. The mean number of consultations per patient was 1.22 $\pm 1.19(0-6)$.
Cardiology and Infectious Disease consultations were more common in the oldest-old group (Table 2).

The most common laboratory investigations performed included a complete blood count (99.5\%), serum electrolyte panel (99.5\%), and blood gas testing venous or arterial (98.9\%). No statistically significant difference was observed between older and oldest-old group in laboratory values $(P>0.05)$. The distribution of laboratory tests of the study population according to age groups is shown in Figure 1.

ED diagnoses and outcomes of patients according to age groups is shown in Table 3. The most common ED diagnoses were COPD exacerbation (32.6\%), pneumonia (31.1\%), HF (26.1\%), and hypertensive pulmonary edema (8.0\%). Mixed cardiac and respiratory causes were detected in $11.6 \%$, whereas exacerbation of COPD with pneumonia was detected in $6.7 \%$ and pneumonia with acute renal failure in $3.8 \%$ of patients. Patients included in the "other group" presented with diverse conditions, such as malignancies $(n=6)$, malignant pleural effusion $(n=3)$, multi-organ failure $(n=3)$, cerebrovascular accidents $(n=3)$, allergic reactions $(n=2)$, urosepsis $(n=3)$, gastrointestinal bleeding $(n=3)$, liver cirrhosis $(n=1)$, hypertension $(n=9)$, warfarin overdose $(n=5)$, hyponatremia $(n=2)$, complete heart block $(n=1)$, pericarditis $(n=1)$, and pericardial effusion $(n=1)$. Of the patients included in this study, $47.3 \%$ were discharged from the ED, $40.9 \%$ were hospitalized (including inpatients and patients who were transferred to other hospitals after stabilization owing to insufficient bed capacity). $6.4 \%(n=42)$ were admitted to wards and $34.5 \%(n=226)$ were admitted to the ICU (Table 3). Among the inhospital admissions, 26 patients were admitted to Internal Medicine, 5 to Thoracic Surgery, 1 to Neurology, and 1 to Urology.

Even though there was no statistical significance between the patient admission rates and the seasonal pattern of admissions, the number of admissions was highest (34.0\%) in winter and 
Table 2. Distribution of comorbid diseases, imaging, treatment, and consultations of the study population according to age groups

\begin{tabular}{|c|c|c|c|c|}
\hline Variables & Total $(n, \%)$ & Older $(n, \%)$ & Oldest-old ( $n, \%)$ & X2 (P) \\
\hline \multicolumn{5}{|l|}{ Comorbid diseases } \\
\hline Hypertension & $487(74.4)$ & $266(54.6)$ & 221 (45.4) & NS* $^{*}$ \\
\hline $\mathrm{COPD}^{\star \star}$ & $315(48.1)$ & $199(63.2)$ & $116(36.8)$ & $10.068(0.002)$ \\
\hline Heart failure & 300 (45.89) & $158(52.7)$ & 142 (47.3) & NS \\
\hline Coronary artery disease & $248(37.9)$ & $142(57.3)$ & $106(42.7)$ & NS \\
\hline Diabetes & $228(34.8)$ & 149 (65.4) & $79(34.6)$ & $10.436(0.001)$ \\
\hline Atrial fibrillation & $143(21.8)$ & $71(49.7)$ & $72(50.3)$ & NS \\
\hline Cerebrovascular accident & $91(13.9)$ & $57(62.6)$ & $34(37.4)$ & NS \\
\hline Malignancy & $82(12.5)$ & $53(64.6)$ & $29(33.4)$ & NS \\
\hline Chronic renal disease & $80(12.2)$ & $39(48.8)$ & $41(51.2)$ & NS \\
\hline Asthma & $29(4.4)$ & $17(58.6)$ & $12(41.4)$ & NS \\
\hline \multicolumn{5}{|l|}{ Imaging } \\
\hline Thorax CT & $369(56.3)$ & $192(52.0)$ & $177(48.0)$ & $7.808(0.005)$ \\
\hline Chest x-ray & $235(35.9)$ & $150(63.8)$ & $85(36.2)$ & $7.394(0.007)$ \\
\hline Abdominal CT & $139(21.2)$ & $67(48.2)$ & $72(51.8)$ & $4.861(0.02$ \\
\hline Echocardiography & $111(16.9)$ & $49(44.1)$ & $62(55.9)$ & $8.715(0.003)$ \\
\hline CT pulmonary angiography & $83(12.7)$ & $48(57.8)$ & $35(42.2)$ & NS \\
\hline Ultrasonography & $62(9.5)$ & $31(50.0)$ & $31(50.0)$ & NS \\
\hline Venous doppler ultrasound & $20(3.1)$ & $10(50.0)$ & $10(50.0)$ & NS \\
\hline \multicolumn{5}{|l|}{ Interventions } \\
\hline Noninvasive ventilation & $218(33.3)$ & $135(61.9)$ & $83(38.1)$ & NS \\
\hline Mechanical ventilation & $93(14.2)$ & $49(52.7)$ & $44(47.3)$ & NS \\
\hline $\mathrm{CPR}^{\mathrm{R}}$ & $32(4.9)$ & $14(43.8)$ & $18(56.2)$ & NS \\
\hline \multicolumn{5}{|l|}{ Treatment } \\
\hline Oxygen (any delivery mode) & 626 (95.5) & $356(56.9)$ & 270 (43.1) & NS \\
\hline Inhaled beta-agonists & $513(78.3)$ & $302(58.9)$ & 211 (41.1) & NS \\
\hline Antibiotics & $307(46.9)$ & $181(59.0)$ & $126(41.0)$ & NS \\
\hline IV diuretics & $288(44.0)$ & $151(52.4)$ & $137(47.6)$ & $3.988(0.04)$ \\
\hline IV nitrates & $131(20.0)$ & $76(58.0)$ & $55(42.0)$ & NS \\
\hline Anticoagulants & $82(12.5)$ & $42(51.2)$ & $49(48.8)$ & NS \\
\hline Inotropes/vasopressors & $74(11.3)$ & $36(48.6)$ & $38(51.4)$ & NS \\
\hline Rate/rhythm control agents & $42(6.4)$ & $24(51.2)$ & $18(42.9)$ & NS \\
\hline \multicolumn{5}{|l|}{ Consultations } \\
\hline Pulmonary diseases & $287(43.8)$ & $162(56.4)$ & $125(43.6)$ & NS \\
\hline Cardiology & $207(31.6)$ & $101(48.8)$ & $106(51.2)$ & $7.890(0.005)$ \\
\hline Internal medicine & $141(21.5)$ & $70(49.6)$ & $71(50.4)$ & NS \\
\hline Anesthesia & $94(14.4)$ & $53(56.4)$ & $41(43.1)$ & NS \\
\hline Infectious diseases & $57(8.7)$ & $24(42.1)$ & $33(57.9)$ & $5.489(0.01)$ \\
\hline Thoracic surgery & $7(1.1)$ & $4(57.1)$ & $3(42.9)$ & NS \\
\hline General surgery & $5(0.8)$ & $2(40.0)$ & $3(60.0)$ & NS \\
\hline Cardiovascular diseases & $3(0.5)$ & $1(33.3)$ & $2(66.7)$ & NS \\
\hline
\end{tabular}

*NS: Nonspesific;**COPD: Chronic obstructive pulmonary disease; \&CPR: Cardiopulmonary resuscitation. 
lowest $(16.0 \%)$ in summer $(x 2=4.976, P=0.174)$. There was no statistically significant between the seasonal admission patterns and age groups of hospitalized patients $(x 2=1.949, \mathrm{P}=0.583)$.

\section{DISCUSSION}

Depending on the methods used for statistical analysis and the patient population, the prevalence of dyspnea as the presenting symptom elderly patients arriving at the ED by ambulance ranges between $2.4 \%$ and $12 \%$ in the available literature $(2,8,9)$. A recent 3-month retrospective study from Taiwan reported that $11.6 \%$ of elderly patients transported to the ED by ambulance presented with dyspnea (9). A study performed by Prekker et al. in the USA reported that $12 \%$ of patients transported to the ED by ambulance presented with dyspnea (2). A large-scale study performed by Kelly et al. in EDs across Asia, Australia, and New Zealand reported that $5.2 \%$ of patients ( $\geq 18$ years) arrived at the ED (ambulatory and by ambulance) with complaints of dyspnea and that only $2.4 \%$ arrived exclusively by ambulance (8). Dundar et al. observed that elderly patients were most commonly admitted to the ED secondary to dyspnea (18.5\%) (6). In the current study, we observed that the prevalence of elderly patients who were transferred to the ED by ambulance with a chief complaint of dyspnea was significantly high at $16 \%$. When compared with other studies, the study which was having the similar findings was Taiwan study. Although our elderly population is less, the reason for the high number of dyspneic elderly patients presenting by ambulance may be due to the more frequent use of emergency services in our country, the higher incidence of chronic diseases in elderly dyspneic patients or the more frequent use of ambulance services by these patients.

Literature review did not reveal any data with regard to the time and day of admission of elderly patients with dyspnea who are transported to the ED by ambulance. Nearly two-thirds of the patients in the current study arrived at the ED during weekdays and between 08.00 and 24.00 hours. Although this finding cannot be directly compared, Dundar et al. reported that $50 \%$ of the elderly patients investigated in their study arrived at the ED between 12.00 and 24.00 hours (6). In contrast, Crilly et al. observed that the highest number of admissions occurred during weekdays between 07.00 and 15.30 hours (10). Demircan et al. reported that the number of admissions was higher during weekdays (11).

Demircan et al. observed that the mean LOS in the ED among patients aged $\geq 85$ years was 3.6 days (11). A study performed by Sir et al. reported that the mean LOS at the ED among patients aged $\geq 70$ years was 216 min (12). A recent study performed by Street et al. in Australia reported that the median LOS of elderly patients at the ED was 4.7 hours (13). In the current study, the median LOS at the ED was 7.6 hours. In the study reported by Street et al., increasing age was associated with a longer LOS in the ED (13). The findings of our study were consistent with those reported by other studies in the literature. In our study, the LOS in the ED was higher in the oldestold than in the older. Inadequate physiological reserves and multiple medicine uses of elderly patients may cause changes in the responses to stress. Therefore, symptoms and physical examination results of these patients may be unclear. For differential diagnosis, further testing and consultation are requested and performed. Excessive workload and lack of knowledge among ED staff may cause delays in accurate assessments of these patients. In conclusion several factors, such as characteristics of the study population, age, ED patient load, number of hospital beds, excessive workload, and experience of ED staff affect the LOS in the ED.

Most patients in the current study had comorbidities, including chronic cardiac and respiratory illnesses, such as hypertension, COPD, $\mathrm{HF}$, and coronary artery disease, which concurs with the findings reported by previous studies $(3$, $4,8,14)$. Only $5.2 \%$ of the patients in this study 
had no comorbidities. A study by Kelly et al., reported that $12 \%$ of the patients investigated had no apparent comorbidities (8). This difference between studies is attributable to the fact that the current study included patients aged $\geq 65$ years, whereas $60 \%$ of the patients in the study reported by Kelly et al.

Non-invasive and mechanical ventilation were more common in our study than in other studies reported in the literature (8). In our view, this finding was attributable to the fact that the patients investigated in the current study were older, with a greater predisposition to life-threatening situations. Thoracic CT and chest radiography were more commonly performed in the older than in the oldest-old patients. These results were in agreement with the ED diagnoses of patients. Most older patients were diagnosed with COPD exacerbation and pneumonia. Additionally, a wide range of laboratory tests were performed in this study. This study highlights that preexisting chronic conditions and old age necessitate the extensive use of healthcare services.

Elderly patients admitted to EDs receive numerous and consultations with various specialties owing to comorbidities and several complex issues observed in this patient population (12). In the present study, 65\% of the patients underwent consultations with various medical specialties. Yenal et al. reported that $59.8 \%$ of elderly patients in the ED underwent consultations and that consultations were more common among patients arriving by ambulance (15). In a study conducted by Loğoglu et al. at a university hospital, the consultations most commonly requested for elderly patients were cardiology, internal medicine and pulmonary disease, respectively (16). Although not statistically significant, we observed that most consultations were with the pulmonary disease, cardiology, and internal medicine departments, respectively. These results were consistent with the ED diagnoses of patients in the current study. Sir et al. reported that the mean number of consultations for 2000 patients $\geq 70$ years, who arrived at the ED with various complaints in an ambulatory state or via ambulance, was $0.47 \pm 0.74$ (12). In the current study, the mean number of consultations per patient was $1.22 \pm 1.19$. This observation is attributable to the fact that elderly patients who arrive by ambulance with complaints of dyspnea often present with have severe clinical comorbidities, particularly respiratory and cardiac conditions.

Previous studies have reported that, dyspnea was most commonly associated with respiratory and cardiac diseases $(2-4,14,17)$. Our study results are reported by the aforementioned studies. The most common disorders associated with dyspnea observed in the ED were COPD exacerbation, pneumonia, HF, and hypertensive pulmonary edema. Hypertensive pulmonary edema was commonly observed in patients included in our study in contrast to the findings of previous studies, which is, attributable to the fact that hypertension was the most common comorbidity in our study population. Dyspnea in elderly patients is often associated with multiple overlapping disorders, such as pneumonia or COPD exacerbation that may precipitate acute HF (18). A significant number of patients presented with such multifactorial etiology of dyspnea. Acute renal failure and anemia were also identified as prominent causes of dyspnea in addition to respiratory, cardiac, and infectious diseases. Patients categorized as the "other group" were diagnosed with a wide range of conditions, including metabolic disorders such as hyponatremia, neurological conditions such as cerebrovascular accidents, and oncologic complications such as malignant pleural effusion, in addition to gastrointestinal bleeding, warfarin overdose, urosepsis, and allergic reactions. The cause of dyspnea remained unclear in a small percentage of patients. All these data are consistent with those reported in the literature $(2$, 8).

A study performed by Kelly et al. reported a high admission rate $(76.4 \%)$ but a lower rate of ICU admissions (5.6\%) in patients with dyspnea 
Table 3. Distribution of emergency diagnoses and outcomes of the study population according to age groups

\begin{tabular}{|c|c|c|c|c|}
\hline Variables & Total $(n, \%)$ & Older $(n, \%)$ & Oldest-old (n,\%) & $\mathrm{X} 2(\mathrm{P})$ \\
\hline \multicolumn{5}{|l|}{ Diagnosis } \\
\hline COPD** & $214(32.6)$ & 141 (65.9) & $73(34.1)$ & $10.712(0.001)$ \\
\hline Pneumonia & $204(31.1)$ & $122(59.8)$ & $82(40.2)$ & NS* \\
\hline Heart failure & $171(26.1)$ & $88(51.5)$ & $83(48.5)$ & NS \\
\hline Hypertensive pulmonary edema & $53(8.0)$ & $35(66.0)$ & $18(34.0)$ & NS \\
\hline COPD + heart failure & $47(7.2)$ & $29(61.7)$ & $18(38.3)$ & NS \\
\hline Pneumonia + heart failure & $46(4.4)$ & $23(50.0)$ & $23(50.0)$ & NS \\
\hline Pneumonia + COPD & $44(6.7)$ & $37(84.1)$ & 7(15.9) & $14.323(0.0001)$ \\
\hline Acute renal failure & $35(5.3)$ & $11(31.4)$ & $24(68.6)$ & $9.695(0.002)$ \\
\hline Lower respiratory tract infection & $30(4.5)$ & $14(46.7)$ & $16(53.3)$ & NS \\
\hline Pneumonia + acute renal failure & $25(3.8)$ & $11(44.0)$ & $14(56.0)$ & NS \\
\hline Coronary artery disease & $20(3.0)$ & $12(60.0)$ & $8(40.0)$ & NS \\
\hline Anemia & $13(1.9)$ & $10(76.9)$ & $3(23.1)$ & NS \\
\hline Pneumonia+UT| $\beta$ & $12(1.8)$ & $4(33.3)$ & $8(66.7)$ & NS \\
\hline Pulmonary embolism & $10(1.5)$ & $5(50.0)$ & $5(50.0)$ & NS \\
\hline Asthma & $9(1.3)$ & $5(55.6)$ & $4(44.4)$ & NS \\
\hline Pneumothorax & $5(0.7)$ & $4(80.0)$ & $1(20.0)$ & NS \\
\hline Other & $44(6.7)$ & $16(36.6)$ & $28(63.3)$ & NS \\
\hline Unclear & $41(6.2)$ & $20(48.8)$ & $21(51.2)$ & NS \\
\hline \multicolumn{5}{|l|}{$\mathrm{ED}^{\&}$ outcome } \\
\hline Discharged & $310(47.3)$ & $180(58.1)$ & $130(41.9)$ & NS \\
\hline Treatment refusal & $55(8.3)$ & $33(60.0)$ & $22(40.0)$ & NS \\
\hline Exitus (ED and in-hospital) & $22(3.3)$ & $11(50.0)$ & $11(50.0)$ & NS \\
\hline \multicolumn{5}{|l|}{ In-hospitalized } \\
\hline Ward & $33(5.0)$ & $20(60.6)$ & $13(39.4)$ & NS \\
\hline Intensive care unit & $37(5.6)$ & $22(59.5)$ & $15(40.5)$ & NS \\
\hline \multicolumn{5}{|l|}{ Transported to other hospitals } \\
\hline Ward & $9(1.3)$ & $5(55.6)$ & $4(44.4)$ & NS \\
\hline Intensive care unit & $189(28.8)$ & $101(53.4)$ & $88(46.6)$ & NS \\
\hline
\end{tabular}

*NS: Nonspesific; ${ }^{* *}$ COPD: Chronico bstructive pulmonary disease; ${ }^{\circledR}$ ED: Emergency department; ${ }^{\beta}$ UTI: Urinary tract infection. 
Figure 1. The distribution of laboratory tests of the study population according to age groups

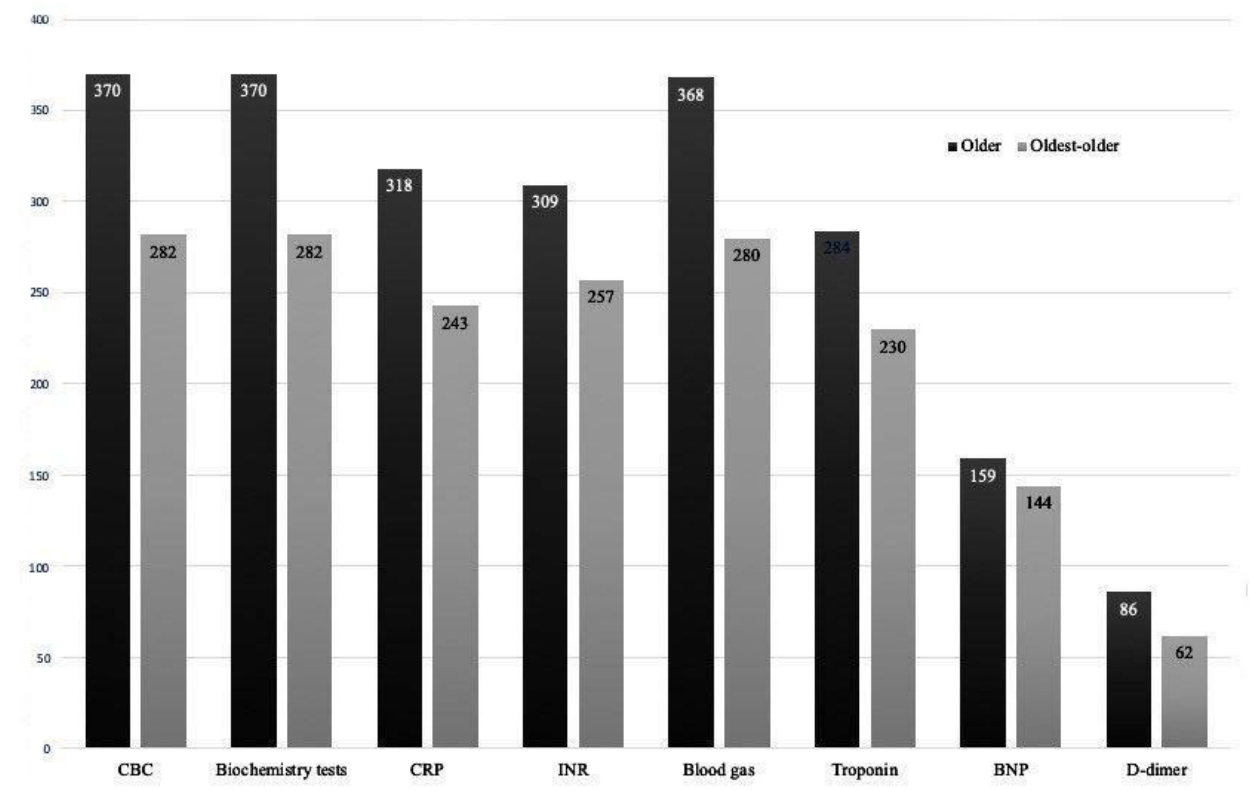

who were transported to the ED by ambulance (4). Another study by the same author that included a larger and different patient population reported that the hospitalization rate was $64 \%$ (including ICU admissions and transfers for admission), and the ICU admission rate was 3.3\% (8). Laribi et al. reported that nearly two-thirds of their study population was hospitalized, and the ward admission rate was higher $(60.6 \%$ vs. $56.4 \%)$ and ICU admission rate was lower (3.4\% vs. $8.4 \%$ ) in the Asia-Pacific region than in Europe (3). The admission rates in our study were lower than those reported in the literature, which is attributable to the differences in the staff capabilities and working conditions between the study settings. Furthermore, the ICU and ward admission rates in the current study were different from those reported by previous studies, which can be explained by the fact that the current study included patients aged $\geq 65$ years, whereas other studies included patients aged $\geq 18$ years. Laribi et al. reported the ICU admission rate was higher in Europe (with a significantly high percentage of elderly patients) than in the Asia-Pacific region (3). The ICU admission rate was 2.5 to 4.6 fold higher in elderly than in younger patients owing to the higher percentage of complex medical issues and comorbidities in the former (19).

Mortality rates reported by previous studies ranged between $5 \%$ and $9.4 \%(3,4,8)$. The inhospital mortality rate in the current study was 3.3\%. However, in our opinion, the actual mortality rate was significantly higher than this figure because more than $50 \%$ of patients who require hospitalization were transferred to other hospitals owing to insufficient bed capacity.

We did not observe any statistical significance between the seasonal admission patterns and age groups of hospitalized patients. Kelly et al. reported that ward admissions and ED visits were the highest in winter (8). We observed similar trend in our study with the highest and lowest hospitalization rates in winter and summer, respectively.

An accurate diagnosis of the cause of acute dyspnea in elderly patients with multiple comorbidities may be challenging for ED physicians, because dyspnea is associated with 
multifactorial etiology, including cardiovascular, respiratory, metabolic, and neuromuscular disorders, malignancy, trauma, infection, or allergic reactions. Morever, it is difficult to attribute dyspnea to a single condition, and acute dyspnea often presents in elderly patients as a combination of multiple diseases, such as pneumonia, which often triggers $\operatorname{HF}(3,4,18)$. In the literature, it is stated that the most common reason for dyspnea is respiratory and cardiac diseases. The differentiation of pulmonary and cardiac causes of dyspnea is a challenging task in emergency dedicine. $(2-4,14,17)$. Our study and previous studies also showed that most patients arrive at the ED following decompensation of chronic respiratory and cardiac disorders such as $\mathrm{HF}$ and COPD; therefore, close monitoring and a multidisciplinary approach are important during the acute phase in the ED, in addition to optimal patient management skills of ED physicians. In addition, appropriate management of this patient group at primary healthcare and outpatient settings may reduce frequent ED admissions and recurrent hospitalizations. Considering the wide range differantial diagnoses in elderly patients with dyspnea, accurately diagnosing this condition may be challenging for emergency physicians. Therefore, emergency physicians should perform a wider range of diagnostic tests ve imaging modalities in this patient group.

There is no rapid and exact diagnosis test to describe the etiology of dyspnea. There are two meta-analysis studies recently carried out for use of BNP in patients presenting to the ED with acute dyspnea. These studies reported that the results recommended the use of BNP to rule out or diagnose HF are inconclusive. Moreover, there are often delays or lack of availability inherent with chest $x$-ray and BNP in patients with acute HF in the ED setting $(20,21)$. Standardization of patient care in chest pain units has improved the quality of patient care $(22,23)$. A similar approach can be performed for patients with a chief complaint of dyspnea in the ED.
The descriptive data regarding elderly patients with a chief complaint of dyspnea, who arrive at the ED by ambulance would benefit ED physicians and hospital administration departments and provide useful information for ED and hospital bed management planning. Our report offers useful insights for optimal planning to train ED personnel for improved management of elderly patients presenting with dyspnea.

Following are the limitations of this study: (a) The retrospective study design is a drawback because access to adequate and reliable data was limited to the capacity of the hospital information management system. (b) This was a single-center study; therefore, our results are not generalizable across all hospitals because working conditions, patient populations, and nature of the hospital vary. (c) This study was based on patient diagnosis; the diagnosis established by ED physicians might have differed from the final diagnosis confirmed in hospitalized patients following detailed assessments. (d) The study included mortality assessment. Owing to the retrospective design, it was not possible to contact patients who were hospitalized at other hospitals, and details regarding survival of these patients were unavailable. Therefore, mortality assessment was limited to evaluation of the survival of patients in the ED. (e) In addition, the lack of control group limits the interpretation of these results.

In conclusion, dyspneic patients present a heterogenous group with various clinical profiles and etiologies for dyspnea throughout the world. The prevalence of elderly patients who arrived at the ED by ambulance with a chief complaint of dyspnea was higher in this study than in other studies reported in the literature. The numerous tests, treatments, and consultations performed, as well as bed requirements tend to drain hospital resources. The results of this study may be leading for future planning of hospitals and healthcare systems. In additions, it may serve as a guide to improve the diagnostic and treatment approaches of ED clinicians. 


\section{REFERENCES}

1. Turkish Statistical Institue. Elderly with statistics, 2019. [Internet] Available from: http://www.tuik. gov.tr/PreHaberBultenleri.do?id=33712. Accesed 18.03.2020. (in Turkish).

2. Prekker ME, Feemster LC, Hough $C L$, et al. The epidemiology and outcome of prehospital respiratory distress. Acad Emerg Med 2014;21:543-50. (PMID: 24842506)

3. Laribi S, Keijzers G, van Meer $O$, et al. AANZDEM and EURODEM study groups. Epidemiology of patients presenting with dyspnea to emergency departments in Europe and the Asia-Pacific region. Eur J Emerg Med 2019 Oct;26(5):345-349. (PMID: 30169464).

4. Kelly AM, Holdgate A, Keijzers G, et al. AANZDEM study group. Epidemiology, prehospital care and outcomes of patients arriving by ambulance with dyspnoea: an observational study. Scand J Trauma Resusc Emerg Med 2016 Sep 22;24(1):113. (PMID: 27658711).

5. Shrestha AP, Shrestha R, Shrestha SK, et al. Prevalence of dyspnea among patients attending the emergency department of a tertiary care hospital: a descriptive cross-sectional study. J Nepal Med Assoc Sep-Oct 2019;57(219):302-306. (PMID: 32329452).

6. Dundar ZD, Ayranci MK. Presenting Symptoms of Older Emergency Department Patients: A SingleCenter Experience of 10,692 Patients in Turkey. Acta Clin Belg 2019 Aug 12;1-6. (PMID: 31402765).

7. World Health Organization. Men Ageing and Health. Geneva: WHO; 1999. P 10. [Internet] Available from: http://whqlib-doc.who.int/hq/2001 WHO_NMH_ NPH_01.2.pdf. Accessed: July 13, 2014.

8. Kelly AM, Keijzers G, Klim S, et al. An observational study of dyspnoea in Emergency Departments: The Asia, Australia, and New Zealand Dyspnoea in Emergency Departments Study (AANZDEM). Acad Emerg Med 2017;24:328-336. (PMID: 27743490).

9. Huang CC, Chen WL, Hsu CC, et al. Elderly and nonelderly use of a dedicated ambulance corps' emergency medical services in Taiwan. Biomed Res Int 2016;2016:1506436. (PMID: 27478825).

10. Crilly J, Chaboyer W, Wallis M, et al. Predictive outcomes for older people who present to the emergency department. Aust Emerg Nurs J 2008;11:178-183. (DOI:10.1016/j.aenj.2008.07.002).

11. Demircan A, Aygencel Bıkmaz SG, Kadı G, et al. Evaluation of the general characteristics of patients aged 85 years and above admitted to a university hospital emergency department. Turk J Med Sci 2017 Nov 13;47(5):1393-1402. (PMID: 29151309).

12. Özcan Sir, Gijs Hesselink, Mara Van Den Bogaert, et al.Risk factors for prolonged length of stay of older patients in an academic emergency department: A Retrospective Cohort Study. Emerg Med Int 2019 May 2;2019:4937827. (PMID: 31186963).

13. Street $M$, Mohebbi M, Berry $D$, et al. Influences on emergency department length of stay for older people. Eur J Emerg Med 2018; 25(4): 242-249. (PMID:28151752).

14. Bilben B, Grandal L, Sovik S. National Early Warning Score (NEWS) as an emergency department predictor of disease severity and 90-day survival in the acutely dyspneic patient - a prospective observational study. Scand J Trauma Resusc Emerg Med 2016; 24:80. (PMID: 27250249)

15. Yenal S, Keser G, Mandiracıoglu A, et al. The determination of the status of emergency department use by elderly patients and of the indicators for ambulance use. Turkish Journal of Geriatrics 2018;21(2):109-17. (in Turkish).

16. Loğoğlu A, Ayrık C, Köse A, et al. Analysis of nontraumatic elderly patient presentations to the emergency department. Tr J Emerg Med 2013; 13(4):171-9. (in Turkish).

17. Mockel M, Searle J, Muller R, et al. Chief complaints in medical emergencies: do they relate to underlying disease and outcome? The Charité Emergency Medicine Study (CHARITEM). Eur J Emerg Med 2013;20:103-8. (PMID: 22387754).

18. Tyler K, Stevenson D. Respiratory Emergencies in Geriatric Patients. Emerg Med Clin N Am 2016;34(1):39-49. (PMID: 26614240).

19. Hamel MB, Davis RB, Teno JM, et al. Older age, aggressiveness of care, and survival for seriously ill, hospitalized adults. Ann Intern Med 1999;Nov 16;131(10):721-8. (PMID:10577294).

20. Lam LL, Cameron PA, Schneider HG, et al. Metaanalysis: effect of B-type natriuretic peptide testing on clinical outcomes in patients with acute dyspnea in the emergency setting. Ann Intern Med 2010; 153:728-35. (PMID: 21135296)

21. Trinquart L, Ray P, Riou B, et al. Natriuretic peptide testing in EDs for managing acute dyspnea: a metaanalysis. Am J Emerg Med 2011; 29: 757- 67.(PMID: 20825895). 
22. Keller T, Post F, Tzikas $S$, et al. Improved outcome in acute coronary syndrome by establishing a chest pain unit. Clin Res Cardiol 2010; 99:149-155. (PMID: 20033995).

23. Furtado MV, Cardoso A, Patricio MC, et al. Influence of implementation of a chest pain unit on acute coronary syndrome outcomes. J Emerg Med 2011; 40:557-564. (PMID: 20022199): 